\title{
Characteristics of Symptomatic Belching in Patients With Belching Disorder and Patients Who Exhibit Gastroesophageal Reflux Disease With Belching
}

\author{
Shin Ok Jeong, ${ }^{1}$ Joon Seong Lee, ${ }^{1 *}$ Tae Hee Lee, ${ }^{1}$ Su Jin Hong, ${ }^{2}$ Young Sin Cho, ${ }^{3}$ Junseok Park, ${ }^{1}$ Seong Ran Jeon, ${ }^{1}$ \\ Hyun Gun Kim, ${ }^{1}$ and Jin-Oh Kim ${ }^{1}$ \\ ${ }^{1}$ Institute for Digestive Research, Digestive Disease Center Soonchunhyang University College of Medicine, Seoul, Korea; ${ }^{2}$ Digestive Disease \\ Center and Research Institute, Department of Internal Medicine, Soonchunhyang University College of Medicine, Bucheon, Gyeonggi-do, Korea; \\ and ${ }^{3}$ Division of Gastroenterology, Department of Internal Medicine, Soonchunhyang University Cheonan Hospital, Soonchunhyang University \\ College of Medicine, Cheonan, Chungcheongnam-do, Korea
}

\section{Background/Aims}

Belching disorder (BD) is clinically distinct from gastroesophageal reflux disease (GERD) with belching. Supragastric belching (SGB) is closely associated with reflux episodes. This study investigates belch characteristics in association with reflux, compared between patients with BD and those who had GERD with belching.

\section{Methods}

Impedance $\mathrm{pH}$ monitoring data from 10 patients with BD and 10 patients with GERD who exhibited belching were retrospectively analyzed. Belches were considered "isolated" or "reflux-related" and acidic/non-acidic. Belch characteristics were compared between patients with $B D$ and those with GERD.

\section{Results}

Symptomatic belches were more frequent in patients with BD than in patients with GERD (median, 160.5 vs 56.0, $P<0.05$ ). SGB was the most common type in both groups; common subtypes comprised "isolated" in patients with BD and "isolated during the reflux period" in patients with GERD. Reflux-related SGB was more common in patients with GERD than in BD ( $78.3 \%$ vs $45.2 \%, P<0.005)$. Both "preceding belching" including the reflux period and acidic SGB were more common in patients with GERD than in BD (31.8\% vs $8.6 \%$ and $38.1 \%$ vs $8.9 \%$, both $P<0.05)$. Supragastric belch number positively correlated with all reflux episodes in patients with GERD (adjusted $R^{2}=0.572, P=0.007$ ).

\section{Conclusions}

$B D$ is characterized by more belching, compared to GERD. SGB is more frequently associated with reflux in GERD than in BD; acidity may be related to GERD. In BD, SGB is typically non-acidic and unrelated to reflux. Distinct SGB characteristics may reflect different pathogenic mechanisms of reflux and associated symptoms.

(J Neurogastroenterol Motil 2021;27:231-239)

Key Words

Electric impedance; Esophageal pH monitorings; Eructation; Gastroesophageal reflux

Received: May 22, 2020 Revised: August 2, 2020 Accepted: August 16, 2020

(a) This is an Open Access article distributed under the terms of the Creative Commons Attribution Non-Commercial License (http://creativecommons. org/licenses/by-nc/4.0) which permits unrestricted non-commercial use, distribution, and reproduction in any medium, provided the original work is properly cited.

*Correspondence: Joon Seong Lee, MD Institute for Digestive Research, Soonchunhyang University College of Medicine, 59 Daesagwan-ro, Yongsan-gu, Seoul 140-743, Korea

Tel: +82-2-709-9691, Fax: +82-2-709-9696, E-mail: joonlee@schmc.ac.kr 


\section{Introduction}

In the clinical setting, belching disorder (BD) is easily distinguished from gastroesophageal reflux disease (GERD) with belching because the sole complaint in patients with $\mathrm{BD}$ is typically bothersome excessive belching, sufficient to impact daily activities and impair social functioning (eg, social isolation and onset of anxiety disorder). ${ }^{1}$ Belches in patients with $\mathrm{BD}$ are usually supragastric, caused by intake of air from the pharynx to the esophagus, followed by rapid expulsion of the air. In contrast, physiologic gastric belching (GB) is induced by a vagal reflex (ie, transient lower esophageal sphincter relaxation [TLESR]). ${ }^{2}$ Supragastric belching (SGB) is often excessive and impacts affected patients' social lives; however, it is regarded as a behavioral disorder. For example, SGB spells may be frequent when affected patients consult their physicians. Notably, belching is also a frequently reported symptom in patients with GERD. ${ }^{3}$ In this group, GB is typical; however, SGB may also occur and is reported by half of patients with reflux symptoms. ${ }^{4,5}$

In a previous study, approximately half of the SGB episodes occurred in close association with reflux episodes in patients who exhibited reflux symptoms and SGB. Thirty percent of all SGB episodes occurred immediately before the onset of a reflux episode; $18 \%$ occurred during the reflux episode. ${ }^{4}$ This finding suggests that SGB underlies gastroesophageal reflux (GER) in some patients with GERD. In contrast, patients with BD typically do not complain of reflux symptoms, although they may experience more frequent SGB than patients with GERD. This indicates differences in SGB between patients with GERD and those with BD; however, the characteristics of belching, including the relationships of SGB with the 2 disorders, have not yet been determined.

This study investigates and compares the characteristics of belching (ie, SGB and GB) in association with GER in patients with $\mathrm{BD}$ and patients who had GERD with belching.

\section{Materials and Methods}

\section{Patients}

Ten patients with $\mathrm{BD}$ and 10 patients with typical GERD symptoms as the main concern and belching, were randomly selected and their clinical data were retrospectively evaluated. All patients had undergone 24-hour multichannel intraluminal impedance and $\mathrm{pH}$ (MII-pH) monitoring, as well as high-resolution manometry, at Soonchunhyang University Hospital (Seoul, Republic of Korea) during the past 5 years. All patients with BD met the Rome IV criteria (B2a: excessive SGB) and all patients with GERD had typical symptoms (eg, heartburn and/or regurgitation) as the main symptom. ${ }^{1}$ Exclusion criteria were incomplete medical records, MII-pH monitoring performed on proton pump inhibitor therapy, previous gastrointestinal tract surgery, and $<5$ belching events on diary during impedance $\mathrm{pH}$ monitoring. The study protocol was approved by the Institutional Review Board of Soonchunhyang University Seoul Hospital (2020-01-016).

\section{Multichannel Intraluminal Impedance and $\mathrm{pH}$ Monitoring and High-resolution Manometry Measurement}

All patients fasted from midnight until the time of measurement. A combined $\mathrm{pH}$-impedance catheter (Sandhill Scientific, Inc, Highlands Ranch, CO, USA) was placed transnasally such that the esophageal $\mathrm{pH}$ electrode was situated $5 \mathrm{~cm}$ above the upper margin of the manometrically defined lower esophageal sphincter (LES). The catheter's 8 electrode rings enabled impedance recording at 6 segments $(3,5,7,9,15$, and $17 \mathrm{~cm})$ above the LES. All patients had discontinued proton pump inhibitor use for at least 7 days previously; they had also discontinued other medications that may affect gastrointestinal motility or acidity for at least 72 hours previously. Otherwise, patients were encouraged to maintain their normal daily activities and usual diet. Event markers on the data logger were used to record the start and end times of symptoms (only start time for belching), meal times, and position changes. The recorded data were analyzed using a dedicated software program (BioView Analysis; Sandhill Scientific, Inc), in conjunction with visual analysis using an appropriate time window and zooming. ${ }^{6}$

High-resolution manometry was performed immediately prior to MII-pH monitoring. A high-resolution manometry catheter (Sierra Scientific Instruments Inc, Los Angeles, CA, USA) was inserted transnasally with the patient in a sitting position. The highresolution manometry protocol included 30 seconds without swallows to assess basal esophagogastric junction pressure and morphology, followed by 10 swallows of $5 \mathrm{~mL}$ of $0.3 \%$ saline solution. ${ }^{?}$

\section{Data Analysis}

Twenty-four hour MII-pH monitoring data were retrospectively analyzed by 2 gastroenterology physicians employed at Soonchunhyang University Hospital. SGB was defined as a rapid rise in impedance ( $\geq 1000 \Omega$ ) moving in an aboral direction, followed by a return to baseline moving in the oral direction. ${ }^{8} \mathrm{~GB}$ was defined as an elevation of intraluminal impedance $(\geq 1000 \Omega)$, including 
pure gas and mixed liquid gas reflux episodes moving in the oral direction and reaching the most proximal impedance recording segment (Fig. 1).

SGB related to GER was classified as preceding, during, or following belching. Preceding belching $(\mathrm{PB})$ was defined as a belch that occurred immediately prior ( $<1$ second) to the onset of the reflux episode, evidenced as a $>50 \%$ reduction in the impedance level. During belching was defined as a belch that occurred during the reflux episode ${ }^{4}$; following belching $(\mathrm{FB})$ was defined as a belch that occurred immediately after the reflux episode ( $<1$ second). Belches not related to reflux were regarded as isolated belching (IB). If $\mathrm{PB}$ occurred during the reflux period (re-reflux), it was classified as during-preceding belching (DPB); during-isolated belching (DIB) was defined as belching that occurred without re-reflux
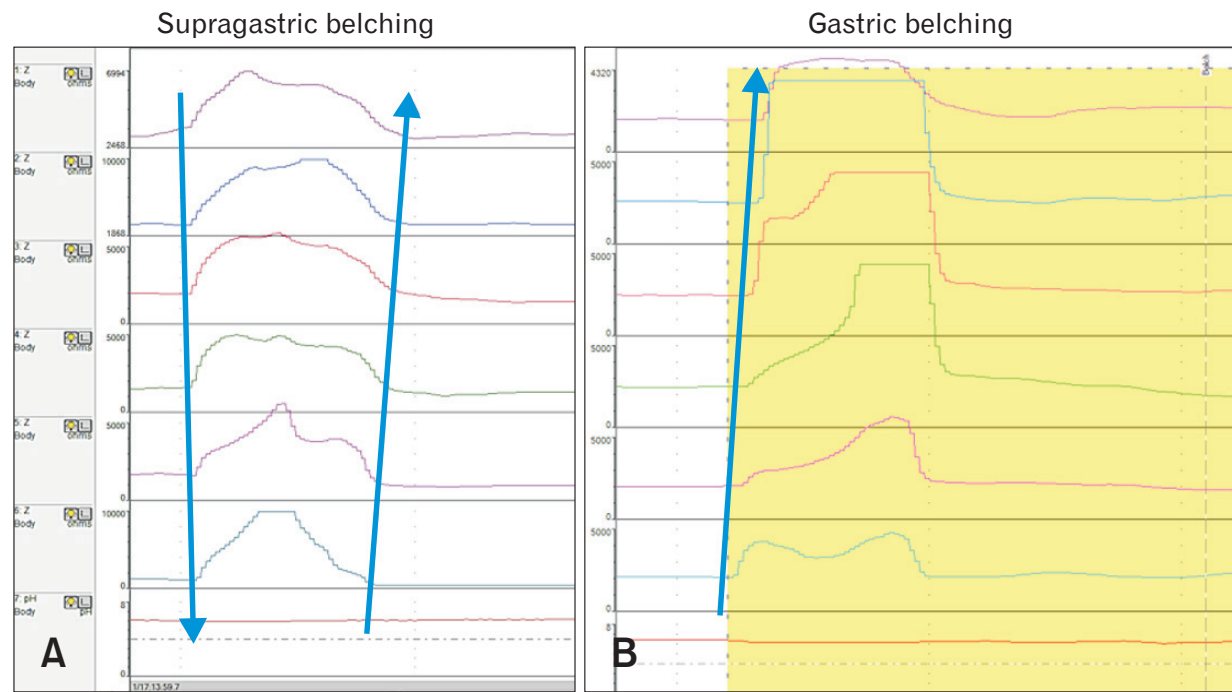

Figure 1. Supragastric belching (SGB) and gastric belching (GB). (A) SGB was defined as a rapid rise in impedance $(\geq 1000 \Omega)$ moving in an aboral direction, followed by a return to baseline moving in the oral direction. (B) GB was defined as an elevation of intraluminal impedance $(\geq 1000 \Omega$ ), including pure gas and mixed liquid gas reflux episodes, moving in the oral direction and reaching the most proximal impedance recording segment. Modified from Bredenoord et al. ${ }^{8}$
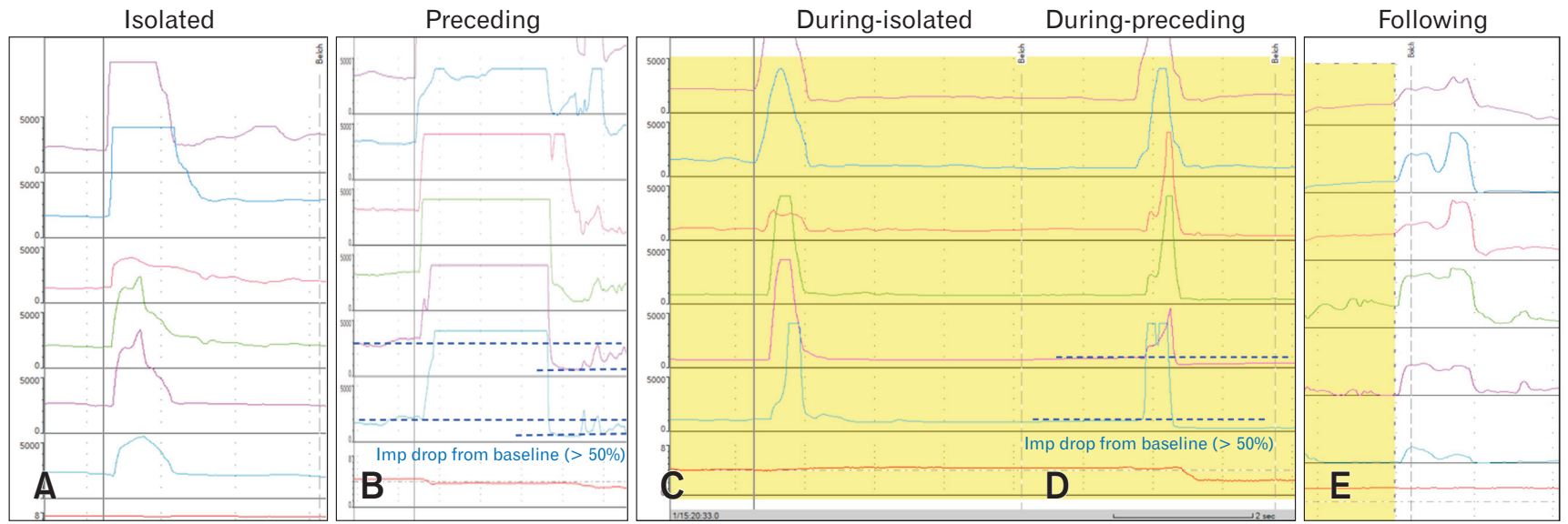

Figure 2. Supragastric belching (SGB) related to reflux. (A) SGB was regarded as "isolated belching" when the belch was unrelated to a reflux event. (B) "Preceding belching" was defined as a belch that occurred immediately prior $(<1$ second) to the onset of the reflux episode, which appeared as a $>50 \%$ reduction in impedance level. (C) "During-isolated belching" was defined as a belch that occurred during the reflux episode but did not induce re-reflux. (D) "During-preceding belching" was defined as a supragastric belch that preceded the during the reflux period (rereflux). (E) "Following belching" was defined as a belch that occurred immediately after the reflux episode ( $<1$ second). Modified from Hemmink et al. ${ }^{4}$ 
during reflux periods (Fig. 2). All reflux episodes were classified as acidic $(\mathrm{pH}<4)$ and non-acidic $(\mathrm{pH}>4)$ reflux, according to the $\mathrm{pH}^{9}{ }^{9}$

Reflux parameters determined by MII-pH monitoring were compared between patients with BD and patients who exhibited GERD with belching. Parameters of interest were as follows: esophageal acid exposure \% time, rate of acidic bolus exposure time, and symptom index $>50 \% .{ }^{10}$ Esophageal acid exposure $\%$ time was defined as the percentage of total time $\mathrm{pH}$ was $<4.0$ units divided by the time monitored. Bolus exposure time was defined as the time from liquid bolus entry ( $50 \%$ down in baseline impedance) to exit (returning to entry level for $\geq 5$ seconds). High-resolution manometry parameters, including hyper-dynamic upper esophageal sphincter (UES) pressure, hyperdynamic UES, ${ }^{11}$ LES pressure, and integrated relaxation pressure, were also compared between the 2 groups of patients.

Other comparisons between patients with $\mathrm{BD}$ and patients who exhibited GERD with belching were total belching/SGB/GB, the $\mathrm{SGB} / \mathrm{GB}$ ratio, the $\mathrm{PB} / \mathrm{DPB} / \mathrm{DIB} / \mathrm{FB} / \mathrm{IB}$ ratio of $\mathrm{SGB}$, and the ratio of acidic/non-acidic SGB/GB. Correlations between GB or SGB and the reflux parameters measured by MII-pH monitoring were also determined in the 2 groups.

\section{Statistical Methods}

Statistical analyses included Student's t-test, the Pearson chisquared test, Spearman's rank test, and linear regression for correlations. Differences were considered significant at $P$-values $<$ 0.05. All analyses were performed using SPSS Statistics version 22.0 (IBM Corp, Armonk, NY, USA). Continuous variables are reported as the mean and standard deviation or median and range; categorical variables are reported as the number and proportion.

\section{Results}

\section{Characteristics of the Patients}

Patients in the BD group were not significantly different from those in the GERD group with respect to age and sex. Median ages were 40.5 (28-77) years in the BD group and 58.5 (36-61) years in the GERD group.

The rate of esophageal acid exposure \% time was not significantly higher in the GERD group than in the BD group, whereas

Table. Characteristics of the Patients

\begin{tabular}{|c|c|c|c|}
\hline Baseline characteristics & Belching disorder $(\mathrm{n}=10)$ & $\operatorname{GERD}(\mathrm{n}=10)$ & $P$-value \\
\hline Age (yr) & $40.5(28-77)$ & $58.5(36-61)$ & NS \\
\hline Gender & 5 female & 6 female & NS \\
\hline \multicolumn{4}{|l|}{ Impedance-pH monitoring data } \\
\hline Esophageal acid exposure $\%$ time & $2.9(0-7.1)$ & $5.2(3.1-11)$ & NS \\
\hline Composite score & $10.1(0.8-25.3)$ & $15.9(10.3-39.9)$ & NS \\
\hline Acidic bolus exposure time & $0.75(0.1-3.2)$ & $2.4(1.5-7)$ & 0.002 \\
\hline Number of liquid reflux & $42(24-100)$ & $67(30-141)$ & 0.028 \\
\hline SI $>50 \%$ for belching & $2(20)$ & $9(90)$ & 0.0055 \\
\hline $\mathrm{SAP} \geq 95 \%$ for belching & $7(70)$ & $10(100)$ & NS \\
\hline SI $>50 \%$ for heartburn or regurgitation & $1(10)$ & $4(40)$ & NS \\
\hline $\mathrm{SAP} \geq 95 \%$ for heartburn or regurgitation & $0(0)$ & $1(10)$ & NS \\
\hline \multicolumn{4}{|l|}{ High-resolution manometry data } \\
\hline UES pressure (mmHg) & $34(9.4-96.3)$ & $49.3(20-72.5)$ & NS \\
\hline Hyper-dynamic UES (pressure differences, mmHg) & $43.85(4.3-94.9)$ & $52.6(18.8-98.3)$ & NS \\
\hline LES pressure (mmHg) & $13.8(6-41.7)$ & $17.6(2.5-40.2)$ & NS \\
\hline Integrated relaxation pressure $(\mathrm{mmHg})$ & $52.6(18.8-98.3)$ & $10.8(1.3-13.6)$ & NS \\
\hline \multicolumn{4}{|l|}{ Chicago classification findings } \\
\hline Ineffective esophageal motility & 5 & 4 & \\
\hline Fragmented peristalsis & 4 & 1 & \\
\hline Absent contractility & 0 & 2 & \\
\hline Hypercontractile esophagus & 1 & 0 & \\
\hline
\end{tabular}

GERD, gastroesophageal reflux disease; NS, not significant; SI, symptom index; SAP, symptom association probability; UES, upper esophageal sphincter; LES, lower esophageal sphincter.

Data are presented as median (range) or $\mathrm{n}(\%)$. 
the rate of acidic bolus exposure time was significantly higher in the GERD group than in the BD group (median [range]: 2.4 [1.57.0] vs 0.8 [0.1-3.2], $P<0.05)$. The proportion of patients with a symptom index $>50 \%$ for belching at the impedance $\mathrm{pH}$ was also significantly higher in the GERD group than in the BD group (median: $90 \%$ vs 20\%, $P<0.05$ ). In manometric diagnosis based on the Chicago classification, BD group represented 5 ineffective esophageal motility, 4 fragmented peristalsis, and 1 Jackhammer esophagus; GERD group represented 4 ineffective esophageal motility, 2 absent contractility, 1 fragmented peristalsis, and 3 normal findings. Hiatal hernia was present in 1 from the BD group and 4 from the GERD group.

Manometric parameters (eg, UES pressure, hyperdynamic UES, LES pressure, and integrated relaxation pressure) did not significantly differ between the BD and GERD groups (Table).

\section{Distribution of Belching}

In the BD and GERD groups, 3247 and 701 symptomatic belches were manually analyzed, respectively. Of these, 68 belches from the BD group and 4 belches from the GERD group could not be classified because of the atypical shape of the respective impedance line (undefined). In addition, 20 belches from the BD group and 3 belches from the GERD group were erroneously recorded on diary (no belches). Thus, 3159 belches from the BD group and 694 from the GERD group were included in additional analyses. SGB and GB were classified as IB and reflux-related belching; reflux-related SGB was further classified as PB, DIB,

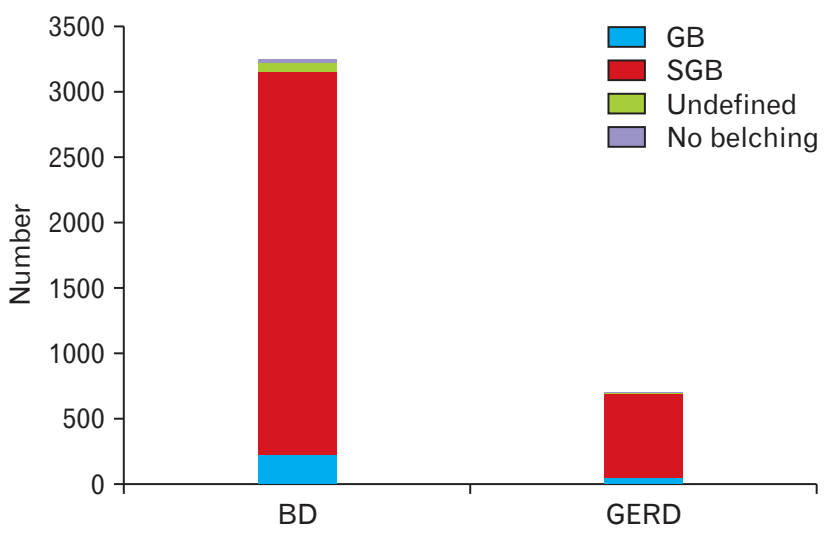

Figure 3. Distribution of symptomatic belching types between belching disorder (BD) and gastroesophageal reflux disease (GERD). The total number of symptomatic belches was significantly higher in the BD group than in the GERD group $(P<0.05)$. The most common type of belching in the 2 groups was supragastric belching (SGB); the ratio of SGB to GB did not differ between the 2 groups.
$\mathrm{DPB}$, and $\mathrm{FB}$, as well as acidic or non-acidic.

The total number of symptomatic belches was significantly higher in the BD group than in the GERD group (median: 160.5 vs 56.0, $P<0.05)$. The most common type of belching in both groups was $\mathrm{SGB}$, which did not significantly differ in terms of proportion between the $\mathrm{BD}$ and GERD groups (median [range]: 93.0\% [35.6-100.0\%] vs 86.8\% [66.7-99.1\%]). Median numbers of supragastric belches were 147.0 (range, 21.0-1647.0) in the BD group and 45.5 (4.0-226.0) in the GERD group. In the BD group, GB comprised $7.2 \%$ of the belches (median [range], 10.5 [0.068.0]), whereas it comprised $7.4 \%(4.0$ [1.0-14.0]) in the GERD group (Fig. 3).

\section{Distribution of Supragastric Belching Subtypes}

SGB subtypes differed in terms of frequency both in the BD group (IB [61.0\%], DIB [33.0\%], DPB and PB [3.0\% each], and FB [0.3\%]) and in the GERD group (DIB [43.2\%], DPB [24.8\%], IB [23.9\%], PB [8.2\%], and FB [0.0\%]). The proportion of GER-related supragastric belches was significantly higher in the GERD group than in the BD group (median: $78.3 \%$ vs $45.2 \%$, $P<0.005)$. PB (PB and DPB) was more frequent in the GERD group than in the $\mathrm{BD}$ group (median: $31.8 \%$ vs $8.6 \%, P<0.05$ ) (Fig. 4).

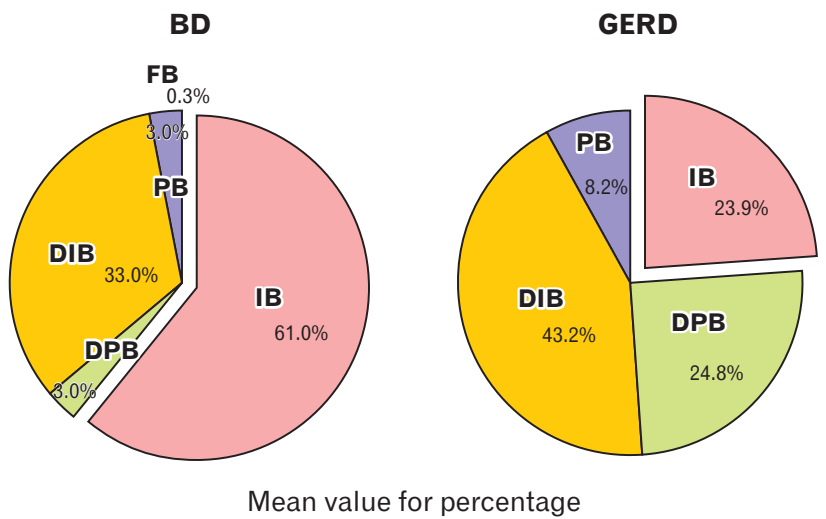

Figure 4. Distribution of the supragastric belching types. Most common belching was isolated belching (IB) in belching disorder (BD), and during belching in gastroesophageal reflux disease (GERD) group. Gastroesophageal reflux related supragastric belching (excluding IB) was significantly higher in GERD group than BD group $(P<0.005)$. Total preceding belching $(\mathrm{PB})$ before and during reflux events was also significantly higher in GERD group than BD group $(P$ $<0.05)$. These finding suggest that supragastric belching may have a role in GERD. FB, following belching; DIB, during-isolated belching; $\mathrm{DPB}$, during-preceding belching. Percentage was a mean value of each individual. 


\section{Acidity of Supragastric and Gastric Belching}

The proportion of acidic belches in SGB events was only $20.0 \%$ in the $\mathrm{BD}$ group, whereas it was $44.0 \%$ in the GERD group.

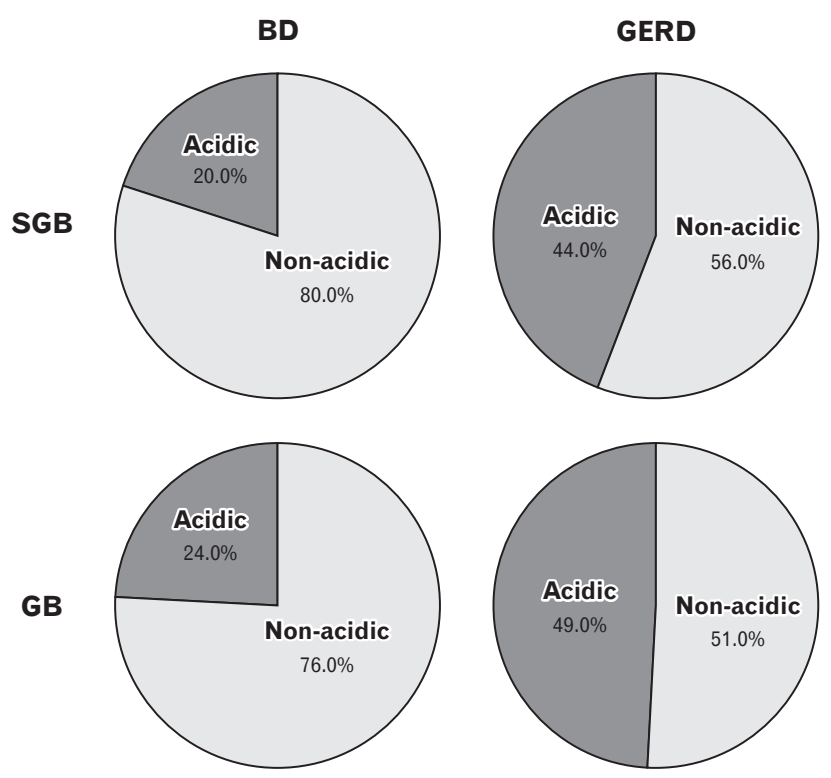

Figure 5. Acidity of supragastric and gastric belches in the belching disorder (BD) and gastroesophageal reflux disease (GERD) groups. In supragastric belching (SGB), the ratio of acidic belches was significantly higher in the GERD group than in the BD group (median, $38.1 \%$ vs $8.9 \%, P<0.05$ ); the ratio of non-acidic SGB was significantly higher in the $\mathrm{BD}$ group. In gastric belching (GB), the ratio of acidic belching was also significantly higher in the GERD group than in the BD group (median: $49.2 \%$ vs $16.9 \%, P<0.05$ ).

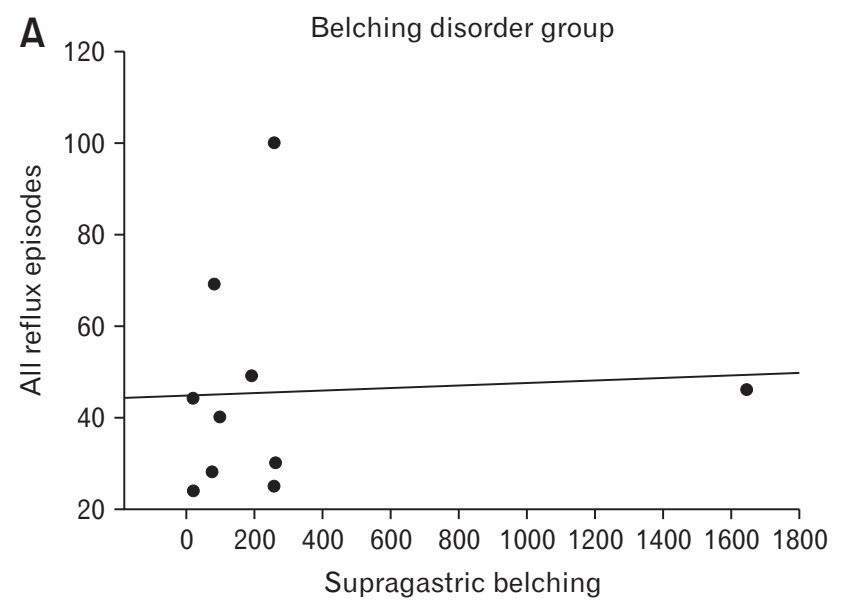

Acidic belching, out of total SGBs, was significantly more common in patients with GERD than in patients with BD (median: $38.1 \%$ vs $8.9 \%, P<0.05)$, whereas non-acidic belching was significantly more common in the BD group (Fig. 5).

Regarding GB events, the proportion of acidic belching was $24.0 \%$ in the BD group, whereas it was $49.0 \%$ in the GERD group. The proportion of acidic belches was also significantly higher in patients with GERD than in patients with BD (median: $49.2 \%$ vs $16.9 \%, P<0.05)$.

\section{Correlation Between Supragastric Belching and Reflux Parameters}

The number of supragastric belches in the GERD group positively correlated with the total number of all reflux episodes (adjusted $\left.R^{2}=0.572, P=0.007\right)$. In the BD group, the number of supragastric belches was not correlated with the total number of reflux episodes (Fig. 6).

Other reflux parameters, including esophageal acid reflux $\%$ time, bolus acid reflux \% time, and bolus all reflux \% time, were not correlated with the number of supragastric belches in either group.

The correlation between the number of gastric belches and the number of all reflux episodes was significantly positive in both groups (GERD group: adjusted $R^{2}=0.436, P=0.022$; BD group: adjusted $\left.R^{2}=0.356, P=0.040\right)$. However, the esophageal acid reflux \% time, bolus acid reflux $\%$ time, and bolus all reflux $\%$ time were not correlated with the number of gastric belches.

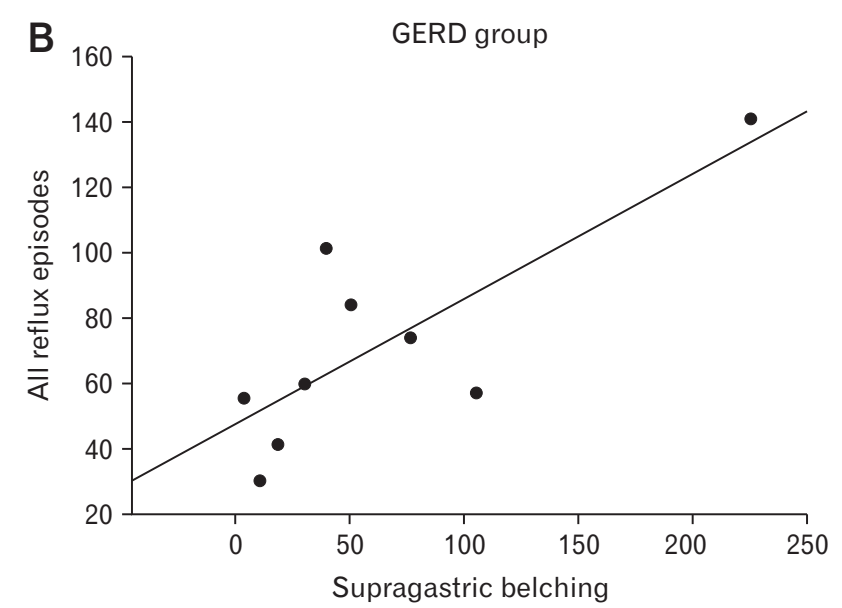

Figure 6. Correlations between supragastric belching (SGB) and all reflux episodes, as determined by impedance pH monitoring. In patients with belching disorder (BD), total reflux episodes were not correlated with the number of supragastric belches (A). However, the number of all reflux episodes was positively correlated with the number of supragastric belches (adjusted $\left.R^{2}=0.572, P<0.05\right)(\mathrm{B})$. 


\section{Discussion}

This is the first study to compare the characteristics of SGB between patients with $\mathrm{BD}$ and patients with GERD. Belching is a common symptom in patients with GERD, but can occur as an isolated symptom. ${ }^{1}$ Belching is present in $40.0-49.0 \%$ of patients with GERD; however, it remains poorly understood. ${ }^{12,13}$ Moreover, the characteristics of belching, including its relationship to GER in patients with $\mathrm{BD}$, have not yet been investigated.

SGB was first demonstrated in patients with excessive belching by measurement of intraluminal impedance. ${ }^{8}$ An elevated incidence of $\mathrm{SGB}$, but not $\mathrm{GB}$, was observed in these patients. In contrast to $\mathrm{GB}$, the incidence of SGB did not significantly increase after patients had eaten a meal. ${ }^{14}$ Previous studies reported that SGB was common in patients with reflux symptoms and that it often preceded a liquid GER episode. ${ }^{4,5}$ These findings suggested that SGB could elicit these episodes in patients with GERD, either at the onset or (more commonly) during a liquid reflux episode, as demonstrated in our study. Because most studies regarding SGB have focused on patients with GERD and there have been no studies of patients with $\mathrm{BD}$, we compared the characteristics of belching in association with GER between patients with BD and patients who exhibited GERD with belching.

Our study showed that belching was more frequent in the BD group than in the GERD group, as expected; however, the SGB/ GB ratio did not differ between the 2 groups. In our patients, SGB was more common than GB in patients with GERD who exhibited belching. However, this finding should be interpreted with caution because patients are more likely to regard SGB as a symptom, rather than GB; we included patients with GERD who reported $>5$ belches per day in their MII-pH symptom diary. In our study, the GERD group represent somewhat high number of belches (median 56.0). This phenomenon may also be due to our exclusion criteria which exclude GERD patients who reported $<5$ belching events during monitoring.

Our results also showed that SGB was more frequently related to GER in patients with GERD than in patients with BD; they also suggested that acidic belching (either GB or SGB) is related to GERD. PB may induce GER ${ }^{4,5}$ and occurred more frequently in patients with GERD than in patients with $\mathrm{BD}$; notably, $\mathrm{PB}$ in patients with GERD occurred most commonly during the GER period. Another important finding of our study was that the frequency of SGB was related to reflux episodes in patients with GERD but not in patients with $\mathrm{BD}$; this was consistent with the results of a recent study in which patients with pathologic acid reflux tended to exhibit a higher number of SGB events. ${ }^{16}$ Other reflux parameters (eg, esophageal acid exposure \% time, bolus acid reflux \% time, and bolus all reflux $\%$ time) were not correlated with the number of supragastric belches and showed only a tendency in the impedance parameters; however, this can be attributed to the small number of patients. Therefore, supragastric belches and the acidity of SGB or GB may contribute to the pathogenic mechanism of GERD; furthermore, $\mathrm{PB}$ may induce GER and re-reflux. In contrast, $\mathrm{SGB}$ in patients with $\mathrm{BD}$ is typically non-acidic $(80.0 \%)$ and may not be related to GER. This suggests differences in the pathophysiologic factors that elicit GER induced by SGB in patients with GERD.

GB is a physiological mechanism that prevents the accumulation of excess gas in the stomach. Previous studies showed that the majority of TLESRs after a meal were associated with transient UES relaxation, which facilitated microburps, regardless of acidity. ${ }^{1718}$ Because GB develops through the mechanism of TLESR, the main mechanism of GERD, it has been regarded as the main type of belching in patients with GERD.$^{19}$ However, our study revealed that SGB is more frequent than GB in patients with GERD, compared to patients with BD. Nonetheless, GB in patients with GERD is characterized by a more acidic gas, relative to patients with BD. As expected, GB positively correlated with total reflux number in both patients with GERD and those with BD.

SGB is provoked by 2 mechanisms, ${ }^{2}$ most commonly involving the air suction mechanism. An initial abrupt contraction of the skeletal diaphragm induces negative pressure in the esophageal lumen. When the glottis is closed, UES relaxation occurs during deep inspiration; subsequently, atmospheric air is sucked into the negatively pressured esophagus. This rapid inflow of air is immediately expelled through UES relaxation, concomitant with elevated intrathoracic pressure that occurs as a result of abdominal straining., ${ }^{2,4} \mathrm{At}$ the esophagogastric junction, the elevated pressure is followed by relaxation, which is induced by abrupt esophageal distension. This may explain why SGB leads to GER. Generally, the UES is relaxed during TLESR, ${ }^{17}$ allowing the air suction type of SGB to occur during the reflux period, as frequently observed in our patients with GERD. The other (much less common) mechanism of SGB comprises air injection, in which air is pushed into the esophagus by the elevated pharyngeal pressure that occurs after voluntary contraction of the tongue base. ${ }^{2}$

Several studies regarding the effect of proton pump inhibitor therapy on belching in patients with GERD have shown that belching events are refractory to such drugs. ${ }^{15}$ DIB in our study is expected to respond well to the proton pump inhibitors usually pre- 
scribed for GERD; IB, PB, and DPB seems to be less responsive. Baclofen, a $\gamma$-aminobutyric acid type $\mathrm{B}$ receptor agonist, causes a slight elevation in LES pressure, thus reducing the numbers of both TLESRs and acid reflux episodes. ${ }^{20-22}$ It may therefore aid in reduction of $\mathrm{SGB}$, including $\mathrm{IB}, \mathrm{PB}$, and $\mathrm{DPB}$, by reducing diaphragmatic contraction and inhibiting TLESR. Diaphragmatic breathing $^{23,24}$ and speech therapy ${ }^{25}$ may also contribute to reductions of all types of SGB, through inhibition of the pathophysiologic mechanism underlying SGB.

Our study had several limitations. First, it used a retrospective design, such that the BD and GERD groups were defined based on chart and exam reviews. Second, the numbers of patients with $\mathrm{BD}$ and those with GERD were low because BD is rare. Studies that include a larger number of patients will yield more precise results.

In summary, BD is characterized by a larger number of belching events than GERD. Most belches in patients with BD and those with GERD are SGB; however, SGB is more frequently related to reflux in patients with GERD than in patients with BD. Acidic belching, either GB or SGB, may be related to GERD. SGB is typically non-acidic and unrelated to reflux in patients with BD. These distinct characteristics of SGB may reflect the pathogenic mechanism of reflux and explain the reflux symptoms. Additional studies will allow the development of strategies to control SGB in patients with GERD and those with BD.

Financial support: This work was supported by the Soonchunhyang University Research Fund.

\section{Conflicts of interest: None.}

Author contributions: Joon Seong Lee conceived the original idea, analyzed raw data from impedance plots, and supervised the project; Shin Ok Jeong analyzed raw data from impedance plots, performed the analytic calculations, and wrote the manuscript; and Tae Hee Lee helped supervise the project and contributed to the interpretation of the results. All authors discussed the results and contributed to the final manuscript.

\section{References}

1. Stanghellini V, Chan FK, Hasler WL, et al. Gastroduodenal disorders. Gastroenterology 2016;150:1380-1392.

2. Kessing BF, Bredenoord AJ, Smout AJ. Mechanisms of gastric and supragastric belching: a study using concurrent high-resolution manometry and impedance monitoring. Neurogastroenterol Motil 2012;24:e573e579.
3. Lin M, Triadafilopoulos G. Belching: dyspepsia or gastroesophageal reflux disease? Am J Gastroenterol 2003;98:2139-2145.

4. Hemmink GJ, Bredenoord AJ, Weusten BL, Timmer R, Smout AJ. Supragastric belching in patients with reflux symptoms. Am J Gastroenterol 2009;104:1992-1997.

5. Kessing BF, Bredenoord AJ, Velosa M, Smout AJ. Supragastric belches are the main determinants of troublesome belching symptoms in patients with gastro-oesophageal reflux disease. Aliment Pharmacol Ther 2012;35:1073-1079.

6. Cho YK, Lee JS, Lee TH et al. The relationship of the post-reflux swallow-induced peristaltic wave index and esophageal baseline impedance with gastroesophageal reflux disease symptoms. J Neurogastroenterol Motil 2017;23:237-244.

7. Park EJ, Lee JS, Lee TH et al. High-resolution impedance manometry criteria in the sitting position indicative of incomplete bolus clearance. $\mathrm{J}$ Neurogastroenterol Motil 2014;20:491-496.

8. Bredenoord AJ, Weusten BL, Sifrim D, Timmer R, Smout AJ. Aerophagia, gastric, and supragastric belching: a study using intraluminal electrical impedance monitoring. Gut 2004;53:1561-1565.

9. Sifrim D, Castell D, Dent J, Kahrilas PJ. Gastro-esophageal reflux monitoring: review and consensus report on detection and definitions of acid, non-acid, and gas reflux. Gut 2004;53:1024-1031.

10. Wiener GJ, Richter JE, Copper JB, Wu WC, Castell DO. The symptom index: a clinically important parameter of ambulatory 24-h esophageal pH monitoring. Am J Gastroenterol 1988;83:358-361.

11. Kwiatek MA, Mirza F, Kahrilas PJ, Pandolfino JE. Hyperdynamic upper esophageal sphincter pressure: a manometric observation in patients reporting globus sensation. Am J Gastroenterol 2009;104:289-298.

12. Bredenoord AJ, Weusten BL, Timmer R, Smout AJ. Characteristics of gastroesophageal reflux in symptomatic patients with and without excessive esophageal acid exposure. Am J Gastroenterol 2006;101:2470-2475.

13. Bredenoord AJ, Weusten BL, Timmer R, Smout AJ. Air swallowing, belching, and reflux in patients with gastroesophageal reflux disease. Am J Gastroenterol 2006;101:1721-1726.

14. Bredenoord AJ. Excessive belching and aerophagia: two different disorders. Dis Esophagus 2010;23:347-352.

15. Kessing BF, Bredenoord AJ, Smout AJ. The pathophysiology, diagnosis and treatment of Excessive belching symptoms. Am J Gastroenterol 2014;109:1196-203.

16. Koukias N, Woodland P, Yazaki E, Sifrim D. Supragastric belching: prevalence and association with gastroesophageal reflux disease and esophageal hypomotility. J Neurogastroenterol Motil 2015;21:398-403.

17. Pandolfino JE, Ghosh SK, Zhang Q, Han A, Kahrilas PJ. Upper sphincter function during transient lower oesophageal sphincter relaxation (tLOSR); it is mainly about microburps. Neurogastroenterol Motil 2007;19:203-210.

18. Gignoux C, Bost R, Hostein J, et al. Role of upper esophageal reflex and belch reflex dysfunctions in noncardiac chest pain. Dig Dis Sci 1993;38:1909-1914.

19. Scheffer RC, Akkermans LM, Bais JE, Roelefs JM, Smout AJ, Gooszen HG. Elicitation of transient lower esophageal sphincter relaxations in response to gastric distension and meal ingestion. Neurogastroenterol 
Motil 2002;14:647-655.

20. Lidums I, Lehmann A, Checklin H, Dent J, Holloway RH. Control of transient lower esophageal sphincter relaxations and reflux by the $\mathrm{GABA}(\mathrm{B})$ agonist baclofen in normal subjects. Gastroenterology 2000;118:7-13.

21. Zhang Q, Lehmann A, Rigda R, Dent J, Holloway RH. Control of transient lower oesophageal sphincter relaxations and reflux by the GABA(B) agonist baclofen in patients with gastro-oesophageal reflux disease. Gut 2002;50:19-24.

22. Cange L, Johnsson E, Rydholm H, et al. Baclofen-mediated gastrooesophageal acid reflux control in patients with established reflux disease.
Aliment Pharmacol Ther 2002;16:869-873.

23. Ong AM, Chua LT, Khor CJ, Asokkumar R, S/O NaMasivayam V, Wang YT. Diaphragmatic breathing reduces belching and proton pump inhibitor refractory gastroesophageal reflux symptoms. Clin Gastroenterol Hepatol 2018;16:407-416, e2.

24. Eherer AJ, Netolitzky F, Högenauer C, et al. Positive effect of abdominal breathing exercise on gastroesophageal reflux disease: a randomized, controlled study. Am J Gastroenterol 2012;107:372-378.

25. Hemmink GJ, Ten Cate L, Bredenoord AJ, Timmer R, Weusten BL, Smout AJ. Speech therapy in patients with excessive supragastric belching-a pilot study. Neurogastroenterol Motil 2010;22:24-28, e2-e3. 